\title{
Motivic Hypercohomology Solutions in Field Theory and Applications in $\mathrm{H}-\mathrm{States}$
}

\author{
Prof. Dr. Francisco Bulnes \\ Correspondence: IINAMEI, Research Department in Mathematics and Engineering, TESCHA, Chalco, Mexico
}

Received: November 15, 2020 Accepted: January 20, 2021 Online Published: January 23, 2021

doi:10.5539/jmr.v13n1p31～URL: https://doi.org/10.5539/jmr.v13n1p31

\begin{abstract}
Triangulated derived categories are considered which establish a commutative scheme (triangle) for determine or compute a hypercohomology of motives for the obtaining of solutions of the field equations. The determination of this hypercohomology arises of the derived category $\operatorname{DM}_{\mathrm{gm}}(k)$, which is of the motivic objects whose image is under $\operatorname{Spec}(k)$ that is to say, an equivalence of the underlying triangulated tensor categories, compatible with respective functors on $\mathrm{Sm}_{k}^{\mathrm{Op}}$. The geometrical motives will be risked with the moduli stack to holomorphic bundles. Likewise, is analysed the special case where complexes $C=\mathbb{Q}(q)$, are obtained when cohomology groups of the isomorphism $H_{e t t}^{p}\left(X, F_{e t}\right) \cong\left(X, F_{N i s}\right)$, can be vanished for $p>\operatorname{dim}(Y)$. We observe also the Beilinson-Soulé vanishing conjectures where we have the vanishing $H^{p}(F, \mathbb{Q}(q))=0$, if $p \leq 0$, and $q>0$, which confirms the before established. Then survives a hypercohomology $\mathbb{H}^{q}(X, \mathbb{Q})$. Then its objects are in $\operatorname{Spec}\left(\mathrm{Sm}_{k}\right)$. Likewise, for the complex Riemannian manifold the integrals of this hypercohomology are those whose functors image will be in $\operatorname{Spec}_{H} \operatorname{SymT}\left(\mathrm{OP}_{L_{G}}(D)\right)$, which is the variety of opers on the formal disk $D$, or neighborhood of all point in a surface $\Sigma$. Likewise, will be proved that $\mathrm{H}^{\vee}$, has the decomposing in components as hyper-cohomology groups which can be characterized as $\mathrm{H}$ - states in $\operatorname{Vec}_{\mathbb{C}}$, for field equations $d \mathrm{da}=0$, on the general linear group with $k=\mathbb{C}$. A physics re-interpretation of the superposing, to the dual of the spectrum $\mathrm{H}^{\vee}$, whose hypercohomology is a quantized version of the cohomology space $H^{q}\left(\right.$ Bun $\left._{G}, \mathcal{D}^{s}\right)=\mathbb{H}_{G[[z]]}^{q}\left(\mathrm{G},\left(\wedge^{\bullet}\left[\Sigma^{0}\right] \otimes \mathbb{V}_{\text {critical }}, \partial\right)\right)$ is the corresponding deformed derived category for densities $\mathrm{h} \in \mathrm{H}$, in quantum field theory.
\end{abstract}

Keywords: Geometrical Motives, Grothendieck Schemes, Motivic Cohomology, Quantized Version of Cohomology, Triangulated Derived Categories

\section{Introduction}

We have some previous results of $\operatorname{PST}(k)$, which is the Universe of all applications, transformations and mappings in Sch, with symmetrizations and triangulations from tensor product structure $\otimes_{R}^{t r}$, [Mac Lane, 1971] to give the category corresponding of the schemes space on $k$ :

$$
\operatorname{Sch}_{k}=\left\{T \in \operatorname{Sch} \mid \mathbb{Z}_{t r}(T)(X \coprod Y)=H_{\text {om }} \text { Cork }_{k}(X \coprod Y, T)\right\}
$$

since $\operatorname{PST}(k)$, is an additive category. A technical lemma affirms that $\mathbb{Z}_{t r}(T)$, is an Étale sheaf [Milne, 1980]. Likewise, if $A$, is an Abelian group then $A \otimes \mathbb{Z}_{t r}(Y)$, for any scheme $Y$, on $k$, is a sheaf in the topology of Zariski, and $A \otimes C_{*} \mathbb{Z}_{t r}(Y)$, is a complex of sheaves.

The additive category PST $(k)$, is the projective special linear group, which is a finite simple group, or automorphism group of the Klein quartic as well as the symmetry group of the Fano plane. This Fano plane is a two-dimensional finite projective plane used for represented roots in finite geometry through symmetrical planar graphs.

Remark 1. Construction of a total tensor product $\otimes^{\mathbb{L}}$, considering extensions of the tensor products $\otimes_{R(A)}^{t r}$, on the category $\operatorname{PST}(k)$, to obtain resolution in the projective sense of infinite sequences of modules of presheaves.

Likewise, we have that the complex $C_{*} \mathbb{Z}_{t r}(Y)$, is not exact in a corresponding succession of these complexes:

$$
Y \rightarrow \mathbb{Z}_{t r}(Y) \rightarrow C_{*} \mathbb{Z}, t r(Y) \rightarrow H_{0} C_{*} \mathbb{Z}, t r(Y)(S),
$$

The last mapping in (2), can be showed that maybe is not surjective, because its co-kernel $H_{0} C_{*} \mathbb{Z}, t r(Y)(S)=\operatorname{Cor}(S, Y) / \mathbb{A}^{1}$ homotopy can be non zero.

Remark 2. $C_{*} \mathbb{Z}_{t r}(Y)$, is a complex of Zariski sheaves. 
Remark 3.We can though in th following diagram

$$
\begin{gathered}
S \\
\downarrow \\
H_{0} \\
\downarrow \rightarrow \mathbb{Z}_{t r}(Y) \rightarrow C_{*} \mathbb{Z}_{t r}(Y) \rightarrow H_{0} C_{*} \mathbb{Z}_{, t r}(Y)(S),
\end{gathered}
$$

Note that $A(q)$, represents the derived sheaf tensor product $\mathbb{Z}(q) \otimes^{\mathbb{L}} A$, since $\mathbb{Z}(q)$, is a flat complex of sheaves. Then for this way arises the necessity of characterize the sheaves through presheaves of derived tensor products starting with tensor products in $\mathrm{Cor}_{k}$.

Then we can define a motivic cohomology groups that will help to define and determine a hypercohomology of the motivic complexes $\mathbb{Z}(q)$, with respect to the Zariski topology.

Definition 1. The motivic cohomology groups $H^{p, q}(X, \mathbb{Z})$, are defined to be the hypercohomology of the motivic complexes $\mathbb{Z}(q)$, with respect to the Zariski topology

$$
H^{p, q}(X, \mathbb{Z}),=\mathbb{H}_{\text {Zar }}^{p}(X, \mathbb{Z}(q)),
$$

If $A$, is Abelian group, we define:

$$
H^{p, q}(X, A),=\mathbb{H}_{\text {Zar }}^{p}(X, A(q)),
$$

Motivic cohomology is well-defined even if the motivic complexes $\mathbb{Z}(q)$, are unbounded complexes because the $X$, in $\mathrm{Sm} / \mathrm{k}$, are finite dimensional [Weibel, 1994]. Likewise, the motivic cohomology is representable in several derived categories.

We have the vanishing theorem.

Theorem 1. For every smooth scheme $X$, and any Abelian group A, we have

$$
H^{p, q}(X, A),=0, \quad p>q+\operatorname{dim} X,
$$

proof. [Mazza, Voevodsky \& Weibel, 2006].

Likewise, the category $\operatorname{Sch}_{k}^{n}$,which is of the functors of presheaves that comply schemes as given in (3), is included as points set:

$$
\operatorname{Sch}_{k}^{n} \subset \mathrm{Sch},
$$

whose functors are constructed considering the Abelian category $S h_{N i s}\left(\operatorname{Cor}_{k}\right)$, and the forgetful functor

$$
S h_{N i s}\left(C o r_{k}\right) \operatorname{PST}(k),
$$

which has a left adjoint $F \rightarrow F_{N i s}$, which is exact and commutes with the forgetful functor to (pre)sheaves on $S m / k$. Likewise, the category $S h_{N i s}\left(\operatorname{Cor}_{k}\right)$, has enough injectives.

Then we can define explicitely the category $\operatorname{Sch}_{k}^{n}$, as:

$$
\begin{array}{r}
\operatorname{Sch}_{k}^{n}=\left\{F \in \operatorname{Sh}_{N i s}\left(\operatorname{Cor}_{k}\right) \mid H_{N i s}^{n}(X, F) \cong \operatorname{Ext}_{S_{N_{N i s}\left(\operatorname{Cor}_{k}\right)}^{n}\left(\mathbb{Z}_{t r}(X), F\right), \forall}\right. \\
\text { smooth } X, \text { and } n \in \mathbb{Z}\}
\end{array}
$$

Likewise our motives category is $\operatorname{DM}_{g m}(\mathbb{Q})=\mathrm{DM}_{g m}(\mathbb{Q}, k)$, which is the derived category of the geometrical motives on the field $\mathbb{Q}$, which involves the quantum fields, or particles of different spin, considering the category of smooth schemes on the numerical field $k$. 
However, requires do it, on complexes $C=V(k)$, when $k=\mathbb{Q}$, where using appropriate functors we have motives with $\mathbb{Q}$ - coefficients. For the appliying of an adequate pre-sheaves motivic cohomology can be identified the transformation images $\operatorname{Tot}_{*}(K)$, which is a full subcategory of $\mathrm{D}^{-}\left(\operatorname{Sh}_{e t}(\operatorname{Cor}(k, \mathrm{R}))\right)$, consisting of complexes with homotopy invariant cohomology sheaves. Likewise, this proves the following result.

Remark 4. $\operatorname{Tot} C_{*}(K)=\left\{K^{\prime} \in \mathrm{D}_{\hat{e} t}^{-}(k, \mathrm{R}) \mid H^{n}\left(K^{\prime}\right)\right.$ is $\mathbb{A}^{1}$-homotopy invariant $\}$.

Theorem 2. The natural functor

$$
\mathcal{L}_{e t} \rightarrow \mathrm{DM}_{e t}^{e f f,-}(k, R),
$$

is an equivalence of triangulated categories if $\mathbb{Q} \subseteq R$.

Proof. [Voevodsky, 2000].

Then as has been demonstrated [MacPherson, Beilinson \& Schechtman, 1987; Bulnes, 2020a; Bulnes, 2020b], the geometrical motives required in our research are result of embed the derived category $\mathrm{DM}_{g m}^{-}(k, R)$, (geometrical motives category) in the $\mathrm{DM}_{e t}^{e f f,-}(k, \mathbb{Z} / m)$, considering the category of smooth schemes on the field $k$.

We consider the following functors. For each $F \in \mathrm{D}^{-}\left(\operatorname{Sh}^{N i s}(\operatorname{Cor}(k))\right)$, there is $L_{\mathbb{A}^{1}} F \in \mathrm{D}_{-}^{e f f}(k)$, the resulting functor is:

$$
L_{\mathbb{A}^{1}}: \mathrm{D}^{-}\left(\operatorname{Sh}^{N i s}(\operatorname{Cor}(k))\right) \rightarrow \mathrm{D}_{-}^{e f f}(k),
$$

which is exact and left-adjoint to the inclusion

$$
\mathrm{D}_{-}^{e f f}(k) \rightarrow \mathrm{D}^{-}\left(\operatorname{Sh}^{N i s}(\operatorname{Cor}(k))\right),
$$

Also the functor (10) descends to an equivalence of triangulated categories. Then can be demonstrated that the operation realised in (10) can be extended to give $\mathrm{D}^{-}\left(\mathrm{Sh}^{N i s}(\mathrm{Cor}(k))\right)$, with the structure of a triangulated tensor category. Then the functor $L_{\mathbb{A}^{1}}$, induces a tensor operation on $\mathrm{D}_{\mathbb{A}^{1}}^{-}\left(\operatorname{Sh}^{N i s}(\operatorname{Cor}(k))\right)$, making that itself $\mathrm{D}_{\mathbb{A}^{1}}^{-}\left(\operatorname{Sh}^{N i s}(\operatorname{Cor}(k))\right)$, is a triangulated tensor category. Likewise, explicitly in $\operatorname{DM}_{-}^{e f f}(k)$, this gives us the functor

$$
m: S m_{k} \rightarrow \mathrm{DM}_{-}^{e f f}(k),
$$

If we consider the embedding theorem, then we can establish the following triangulated scheme

$$
\begin{gathered}
\mathrm{Sm}_{k} \longrightarrow \mathrm{DM}_{g m}^{e f f}(k) \\
m \searrow \uparrow \mathrm{Id} \\
\mathrm{DM}_{g m}^{e f f}(k)
\end{gathered}
$$

which has implications in the geometrical motives applied to bundle of geometrical stacks in mathematical physics. The principal goal of this research is apply a diagram starting on a version of the category $S m_{k}$, on projective motives to obtain a category of Grassmannians, $\operatorname{Gr}(\mathrm{Vec})$, which can be recovered by elements of the Galois group $G_{M o t}=\operatorname{Aut}^{\otimes} H_{B}^{*}(X, \mathbb{Q})$, considering the Grassmannian $\mathbb{G}_{m}$.

This space is a motivic Galois group on $\operatorname{DMT}(k)$, which is a full subcategory of the Tate category of motives $\operatorname{TM}(k)$, then its hypercohomology is a corresponding to the cosmic Galois group, and corresponds to the solution for the field equations.

\section{Some on Derived Categories in Field Theory}

In the derived geometry we work with structures that must support $R$ - modules whose characterizations should be most general to the case of singularities, where is necessary to use irregular connections, if was the case in [Bulnes, 2013].

The generalizations of connections through pre-sheaves defined by derived tensor products with certain special properties, as can be the Étale sheaves, are necessary to establish the triangulated tensor structure of the corresponding derived categories. These are useful in schemes of categories for solutions in the hypercohomology context considering the 
knowledge of the spectral theory of the cycle sequences in motive theory that searches the solution of the field equation even with singularities of the complex Riemann manifold.

We can demonstrate that $\otimes_{\mathbb{L}, e ́ t}^{t r}$, induces a tensor-triangulated structure to a derived category more general than $\mathrm{D}^{-} R(\mathcal{A})$, as for example, $\mathrm{DM}_{e t t}^{e f f^{-}}(k, \mathbb{Z} / m)$, which is our objective. In this case, we want geometrical motives, where this last category $\mathrm{DM}_{e t}^{e f f^{-}}(k, \mathbb{Z} / m)$, can be identified for the derived category $\mathrm{DM}_{g m}^{-}(k, R)$.

We consider $A$, and $B \in \mathcal{A}$, where $\mathcal{A}$, is a category as has been defined before.

We have that $\mathrm{Ab}\left(\mathrm{CRing}_{A / / B}\right) \cong \operatorname{Mod}_{B}$, [Bulnes, 2020a; 2 Bulnes, 2020b]. Then a hypercohomology as given to $d \mathrm{da}=0$, [Bulnes, 2017a] can be obtained through double functor work $A \rightarrow B \rightarrow B$, through an inclusion of a category $\operatorname{Mod}_{B}$, in CRing $_{A / / B}$. Likewise, $\operatorname{Mod}_{B} \operatorname{CRing}_{A / / B}$, is defined by $X \mapsto \Omega_{X / A} \otimes_{X} B$. In particular, the image of $A \rightarrow B \rightarrow B$, under this functor is $B \mapsto \Omega_{X / A}$. As important fact is necessary consider that the derived tensor product is a regular tensor product [Mazza, Voevodsky, \& Weibel, 2006]. Then the adjunction of categories $\mathrm{Ch}(B)_{\geq B} \longleftrightarrow s \mathrm{CRing}_{A / / B}$, induces an adjunction to level of homotopy categories [Mazza, Voevodsky, \& Weibel, 2006].

Remark 5. In the context of the differential operators algebra we can give the commutative rings diagram:

$$
\begin{array}{cccc}
h \in H^{0}\left(T^{\vee} \text { Bun }_{G}, \mathcal{D}^{s}\right) & \stackrel{d}{\longrightarrow} H^{1}\left(T^{\vee} \text { Bun }_{G}, O\right) \stackrel{\cong}{\longrightarrow} \Omega^{1}[\mathrm{H}] \\
\cong \downarrow & \cong \Phi \mu \downarrow & & \downarrow \pi \\
a \in \mathbb{C}\left[O p_{L_{G}}\right] \stackrel{d}{\longrightarrow} & \Omega^{1}\left[O_{O p_{L_{G}}}\right] & \stackrel{d}{\longrightarrow} & C \times B
\end{array}
$$

where $\mathrm{H}^{\vee}=\operatorname{Spec}_{H} \operatorname{SymT}\left(\mathrm{Op}_{L_{G}}(D)\right)$. The second differential operator $d$, in the equations $d \mathrm{da}=0$, comes from the research realized on a meromorphic extension of one flat connection given through a Hitchin construction which satisfies the commutative cocycles diagram to the category $M_{\mathcal{K}_{\mathcal{F}}}(\hat{g}, Y)$ [Bulnes, 2017a].

Finally, we can define the cotangent complex required in derived geometry and QFT, starting with

$$
\operatorname{Mod}_{B} \operatorname{CRing}_{A / / B}
$$

Definition 2. The cotangent complex $\mathbb{L}_{A / B}$, is the image of functor $A \rightarrow B \rightarrow B$, under the left functor of the Kahler differentials module $M \otimes_{R(\mathcal{H})}^{\mathbb{L}}$. Likewise, if $P \bullet \rightarrow B$, be a free resolution then

$$
\mathbb{L}_{A / B}=\Omega_{P_{\bullet} / A} \otimes_{P_{\bullet}} B,
$$

The cotangent complex as defined in (15) lives in the derived category $\operatorname{Mod}_{B}$. We observe that choosing the particular resolution of $B$, then $\Omega_{P_{\bullet} / A}$, is a co-fibrant object in the derived category $\operatorname{Mod}_{P_{\bullet}}$, which no exist distinction between the derived tensor product and the usually tensor product.

\section{Main Result}

Theorem 3. (F. Bulnes). Suppose that $\mathbb{M}$, complex Riemannian manifold with singularities. Let be $X$, and $Y$, smooth projetive varieties in $\mathbb{M}$. We know that solutions of the field equations $d \mathrm{da}=0$, are given in a category $\operatorname{Spec}\left(S m_{k}\right)$, and solution context of the quantum field equations for $d \mathrm{da}=0$, are defined in hyper-cohomology on $\mathbb{Q}$ - coefficients from the category $S m_{k}$, defined on a numerical field $k$, considering the derived tensor product $\otimes_{e t}^{t r}$, of pre-sheaves. Then the following triangulated tensor category scheme is true and commutative:

$$
\begin{gathered}
\mathrm{DQFT} \\
i \measuredangle \quad \searrow F \\
\operatorname{MD}_{g m}(\mathbb{Q}) \longleftrightarrow \operatorname{MD}\left(O_{Y}\right)
\end{gathered}
$$

Proof. [Bulnes, 2020a].

Remark 6. Singular projective varieties useful in quantization process of the complex Riemannian manifold. The quantization condition of compact quantizable Kehler manifolds can be embedded into projective space. 


\section{Motivic Hypercohomology Solutions to Quantum Field Theory: Oscillations of H-States in the Space-Time}

For the gravitation case, the quantum field theory also can establish that the motives must be objects whose triangulated category of geometrical motives is the category $\mathrm{DM}_{g m}(k, R)$, or written simply as $\mathrm{DM}_{g m}(k)$.

This is defined as usually is done, formally inverting the functor of the Tate objects (are objects of a motivic category called Tannakian category) $\mathbb{Z}(1.1)$, to be image of the complex $\left[\mathbb{P}^{1}\right] \rightarrow[\operatorname{Spec}(k)]$, where the motive in degree $p=2,3$, will be $m(p)=m \otimes \mathbb{Z}(1)^{\otimes_{P}}$, or to any motive $m \in \mathrm{DM}_{g m}^{e f f}(k), \forall p \in \mathbb{N}$.

Remark 7. Let $\mathrm{MT}(\mathbb{Z})$, denote the category of mixed Tate motives unramified over $\mathbb{Z}$. It is a Tannakian category with Galois group $\mathrm{Gal}_{M T}$. The Tannakian category is obtained for the inverting of the objects $-\otimes \mathbb{Z}$.(1).

Note. Remember that a scheme is a mathematical structure that enlarges the concept of algebraic variety in several forms, such as taking account of multiplicities. The schemes can to be of a same algebraic variety different and allowing "varieties" defined over any commutative ring. In many cases, the family of all varieties of a type can be viewed as a variety or scheme, known as a moduli space.

Likewise, considering the full triangulated subcategory of the Tannakian category [Saavedra-Rivano, 1972] generated for Tate motives, is available the Beilinson-Soule vanishing theorem [Saavedra-Rivano, 1972] and for the case of the numerical field $k=\mathbb{K}$, we have solutions of the hypercohomology corresponding to the representation of the cosmic Galois group:

$$
\operatorname{Ext}_{\mathrm{DM}(\mathbb{K})}^{1}(\mathbb{Q}(0), \mathbb{Q}(n))=K_{2 n-1}(\mathbb{K}) \otimes \mathbb{Q},
$$

and for the second functor $\operatorname{Ext}_{\mathrm{DM}(\mathbb{K})}^{2}(\mathbb{Q}(0), \mathbb{Q}(n))=0$. The group $K_{2 n-1}(\mathbb{K})$, come from the Quillen's algebraic $K$ - theory [Quillen, 1973].

Remark 8. $K_{2 n-1}(\mathbb{K}) \otimes \mathbb{Q},=\mathrm{H}_{\bullet}(G L(n, k))$, is the linear group of entries in $k$.

Then in this case is possible to extract from the triangulated tensor category a Tannakian category [Saavedra-Rivano, 1972] (as has been mentioned), which we call $\mathrm{TM}_{\text {mix }}(\mathbb{K})$, of mixed Tate motives whose fiber functor $\omega$, (which is a connection) for $\mathbb{Z}$ - graded $\mathbb{Q}$ - vector spaces such that for all $M$, an element of a tensor derived category, we have:

$$
M \mapsto \omega(M)=\oplus_{n} \omega_{n}(M),
$$

with

$$
\omega_{n}(M)=\operatorname{Hom}\left(\mathbb{Q}(n), \operatorname{Gr}_{-2 n}^{w}(M)\right),
$$

where $\mathrm{Gr}_{-2 n}^{w}(M)=W_{-2 n}(M) / W_{-2(n+1)}(M)$, is the graded structure associated to the finite increasing weight filtration $W$.

Likewise, the Grassmann manifold establish components of mass determined by the special functions calculated in each Grassmannian defined for each weight [Morel \& Voevodsky; 1999]. This can suggest a decomposing of functions, which can be states on the connections, obtained of the tensor derived products of the corresponding geometrical derived categories. Now, these states must come from of some field observable in physics. However, the relation between connections (elements of a motivic sheaf) with functions (that can be observables) must be relevant to determine $\mathrm{H}$ - states considering the field corresponding ramifications. Likewise, we have the following result to the respect.

Remark 9. Usually, in the special functions are involving $\zeta$ - functions that determine periods for Tate motives.

Theorem 4. (Function-sheaf correspondence). We have a natural bijection of sets

$$
\left.\operatorname{Hom}\left(H\left(\mathbb{F}_{q}\right)\right), R_{l}(\mathbb{Q})\right) \longleftrightarrow\{l-\text { adic characer sheaves on } H\} \text {, }
$$

where $\mathbb{F}_{q}$, are the tale sheaves category, and $H$, is a commutative group scheme of finite type over $\mathbb{F}_{q}$ with $p \neq l$.

Proof. [Shin, 2005].

Remember that a $l$-adic character sheaf is a character of $E$ tale sheaf where the number $l-$, is a prime number different from the characteristic of $k$. In this case the corresponding cohomology has coefficients in $\mathbb{Z} / l^{n} \mathbb{Z}$.

Remark 10.The term motive of $X$, means "the $l$ - adic cohomology of $X$, with $\mathbb{Q}_{l}-$ coefficients and a continuous $G_{\mathrm{k}}-$ action on it". (The action $G_{\mathrm{k}}-$, is through the motivic Galois group of $X$. 
Remark 11. Theorem 3.18 (Grothendieck). The category of finite Étale schemes over $k$, is equivalent to the category of finite sets with continuous $G_{\mathrm{k}}-$ action. Here $G_{\mathrm{k}}$, is a Galois group whose corresponding representation of the complex variety $X$, is the spectrum $S$ speck.

Remark 12. l- Adic cohomology is a cohomology theory on corresponding varieties which is constructed as an inverse limit of Étale cohomology over different coefficients. This conforms a Weil cohomology theory.

A fundamental fact is that any commutative group scheme over a field $k$, is always an extension of an Abelian variety by an affine group $k$ - scheme. In particular, this is true for the algebras of quantum groups. Likewise, without loose of the meaning of $H$, we can consider to $H$, as an Abelian variety. Then, $H$, as commutative group scheme of a curve, can be the Picard group of a curve. Then if we consider this Picard group of a curve as the Picard variety of a curve $C$, defined for

$$
\mathbb{M}=\operatorname{Pic}(C),
$$

then the Hecke functor is the mapping defined as the integral transform

$$
\Phi^{1}: D_{C o h}(\operatorname{Pic}(C), \mathcal{D}) \rightarrow D_{C o h}(C \times P i c(C), \mathcal{D}),
$$

to $D$ - modules on ${ }^{L}$ Bun. Using the classical limit conjecture is had the equivalence through the interpretation of Higgs sheaves, given in the category $D_{\mathrm{Coh}}\left({ }^{L} \mathrm{Higg}_{0}, O\right)$, which can be extended to the corresponding Langlands correspondence $\mathfrak{c}$, of the "quantum" sheaves given by $\mathfrak{c}=$ quant $_{\text {Bun }} \circ \Phi \circ$ quant $_{C}^{-1}$, where $\Phi$, is the Fourier-Mukai transform that we need. Then as integral, we have the integral transform composition [Milne, 1980], $\mathfrak{c} \circ \Phi^{\mu}={ }^{L} \Phi^{\mu}$, which is solution of the field equations Isom $d \mathrm{~h}=0$, where $\mathrm{h}$, are the mentioned cotangent vectors (Higgs fields). These are the applications we want give to quantum gravity. We want obtain a spectrum in the dual $T^{\vee} \mathrm{Bun}_{G}$, considering the triangle given in (13), whose geometrical motives will be stacks of holomorphic bundles.

Remark 13. In a physical context (could be taken $\mathbb{M}=\operatorname{Pic}(C)$, where $\mathbb{M}$, is the space-time) represents a trace of particles in the symplectic geometry that can be characterized in a Hamiltonian manifold.

Theorem 5 (F. Bulnes). $H^{*}(G L(n, k))$, has the decomposing in components $H^{i}(X)$, that are hyper-cohomology groups corresponding to solutions as $H$ - states in $\operatorname{Vec}_{\mathbb{C}}$ for field equations $d \mathrm{da}=0$.

Proof. We consider the triangle for all $X \in \mathrm{SmProj}_{k}$ :

$$
\begin{aligned}
\operatorname{SmProj}_{k} & \stackrel{m}{\longrightarrow} \operatorname{MT}(k) \\
\searrow \quad & \uparrow \operatorname{Real}(-)
\end{aligned}
$$

GrVect

Then a subcategory of GrVect, can be recovered by elements of the Galois group $G_{m o t}=\operatorname{Aut}^{\otimes} H_{B}^{*}(X, \mathbb{Q})$, considering the Grassmannian $\mathbb{G}_{m}$, as the motivic Galois group (which is equivalent to that certain functors $W_{n}$, from $\mathrm{Gr}_{-2 n}^{w}(M)$, on $\operatorname{DTM}(k)$ (which were induced by the proper tensor and duality structure from the category $\operatorname{DTM}(k)$ ), are the weights filtration of certain Hodge structure "Variation of Hodge Structure" [Griffiths, 1968]). Then if we consider the category $\operatorname{DTM}(k)$ as the derived category $\left.\mathrm{D}^{b} \mathrm{TM}(k)\right)$, which is a full subcategory of the Tate category of motives $\mathrm{TM}(k)$, then its hyper-cohomology is a corresponding to the cosmic Galois group, as given in (17), but with the Abelian structure of the category DTM $(k)$. Then a derived category whose objects can be the geometrical objects, but studied in DQFT (theorem 3) must be of the type

$$
\operatorname{Hom}_{\operatorname{DMT}(k)}(\mathbb{Q}(0), \mathbb{Q}(q)[p])=\operatorname{Ext}_{\mathrm{DMT}(k)}^{p}(\mathbb{Q}(0), \mathbb{Q}(q)),
$$

which is zero for $p<0$. Likewise, from (24) we can find a relation between vanishing theorem of Beilinson-Soulé and the structure of the triangulated category $\operatorname{TM}(k)$. Likewise considering a Tannakian category with mixed Tate objects, we have the following triangulated category

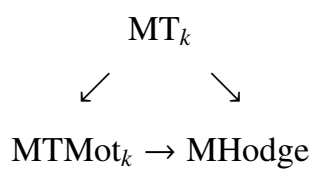


Here, MTMot $_{k} \rightarrow$ MHodge, is exact and faithful. As the weights filtration is of certain Hodge structure, of fact, is a mixed Hodge structure, [Deligne, 1994] then its filtration induces a pure Hodge structure of weight $w$ on $G r_{w}^{W} W_{w} H / W_{w-1} H$, where for our particular case we have induced functors $W_{n}$, on $\operatorname{DMT}(k)$, and taking the associated graded with respect to $W$, then induces faithful exact functors to $\mathbb{Q}$ - vectors space given by

$$
\mathrm{Gr}_{*}^{W}: \mathrm{TM}(k) \rightarrow \mathrm{Vec}_{\mathbb{Q}},
$$

Remark 14. Here the image space MHodge, is a mixed Hodge structure.

Then each motive $M$, can be expressed as $\omega(M)$, which is direct sum of $\omega_{n}(M)$, which has images as the Grassmannians $\mathrm{Gr}_{-2 n}^{w}(M)$. For realization of motives we have:

$$
\operatorname{MT}_{k} \rightarrow \operatorname{GrVec} m(X) \mapsto H^{*}\left(X_{k}, \mathbb{Q}\right),
$$

where in the right extreme we have that are corresponded Grassmannians with groups of the Weil cohomology, Likewise, if we have the decomposing (18) then in the Weil cohomology context we give

$$
H^{*}(\omega(M))=\oplus_{n} H^{q}\left(\omega_{n}(M)\right)
$$

where considering (19) and the hypercohomology determined for solutions in the theorem 3, we have that $q=0$, or $q=1$. Likewise, all cotangent vectors due the hypercohomology has an image in the category of spaces $\operatorname{Vec}_{\mathbb{Q}}$.

Remark 15. Then we have that (26) takes the form $H^{*}(\omega(M))=\oplus_{n} H^{0}\left(\omega_{n}(M)\right)=\oplus_{n} H^{0}\left(\omega_{C}^{n}\right)$, in a Hopf algebra.

By the theorem 4, and having the identification $\overline{\mathbb{Q}}_{1} \rightarrow \mathbb{C}$, (which is possible for the $E$ tale sheaves choosing), all character maps, for example $\Phi$, of a given commutative Hopf algebra $\mathrm{H}$, can arise as an appropriate l-adic character sheaves on $\mathrm{H}$.

Remark 16.Hopf algebras are originated in algebraic topology and are related to the $\mathrm{H}$ - space concept, in group scheme theory, in group theory. Its applications more interesting arise in quantum field theory.

Likewise, if we consider the complex group:

$$
G(\mathbb{C})=\operatorname{Hom}(\mathrm{H}, \mathbb{C})
$$

for this algebra $\mathrm{H}$ and all global functors on $T^{\vee} \mathrm{Bun}_{G}$, are lifted from the basis:

$$
H^{0}\left(T^{\vee} \mathrm{Bun}_{G}, O\right) \cong \mathbb{C}[\mathrm{H}]
$$

Then can be defined solutions in $\Omega^{1}[\mathrm{H}]$, due to that, we need solutions for $d \mathrm{da}=0$, as cotangent vectors. Then need the lifting of the diagram given in the Remark 5, and obtain $\forall \mathrm{h} \in H^{0}\left(\Sigma, K_{\Sigma}^{\otimes 2}\right)$, such that $d \mathrm{~h}=0$. Likewise a Hitchin's construction had that be extended to deformations of stacks $T^{\vee} \operatorname{Bun}_{G}$, and $T^{\vee} B_{L_{G}}$, where there is an extended Penrose transform such that its kernel set has as elements the fields $\mathrm{h}$, with Isom $d \mathrm{~h}=0$, in the hyper-cohomology [Bulnes, 2017a].

Remark 17. We have considered as integrals space the cohomology spaces $H^{\bullet}\left(\mathrm{H}^{\vee}, \Omega^{\bullet}\right)$, inside the cuasi-coherent category given by $M_{\mathcal{K}_{\mathcal{J}}}\left(\mathrm{H}^{\vee}, Y\right)$, which carry us to the ramification problem.

Likewise, we have $H^{2}\left(\operatorname{Bun}_{G}\left(\Sigma,{ }^{L} \mathrm{G}\right), \mathbb{C}\right) \cong \Omega^{1}[\mathrm{H}]$ (where the second differential operator is defined as $\left.d: \Omega^{1}[\mathrm{H}] \rightarrow C \times B\right)$ ) that for the case (21) we obtain $\mathrm{H}^{\vee}$, which is its deformation or spectrum, whose general integral is the extended Hitchin base [Bulnes, 2016; Donagi, \& Pantev, 2010]

$$
\mathcal{H}=H^{0}\left(\omega_{C}\right) \oplus H^{0}\left(\omega_{C}^{\otimes 2}\right) \oplus \cdots \oplus H^{0}\left(\omega_{C}^{\otimes n}\right),
$$

whose hypercohomology has an image in the category of spaces $\operatorname{Vec}_{\mathbb{C}}$.

Example 4. 1 [Bulnes, 2018]. We can consider an Abelian Hodge theory approach and we use $\operatorname{Loc}_{L_{G}}(C)$, the curve of its ramification of the corresponding Langlands data $(\mathrm{M}, \delta)$, where $M=\mathrm{Bun}_{\mathrm{Higgs}}$, and

$$
M \mapsto M \otimes \Omega_{H^{1} g g s^{\prime}}^{1}
$$


(which is $\chi \mapsto \Omega_{\chi / A} \otimes_{\chi} B$ defined in the section 2, to cotangent complex). The differential field equation is

$$
\square \nabla \phi=0,
$$

where for certain conditions $\nabla \phi=\mathrm{h}$, further (33) can be equivalent for certain field interaction (for 2 and 3-dimensional flat spaces) to $\nabla \mathrm{h}=0$, (Isom $d \mathrm{~h}=0$, or its Hitchin-mapping equivalent equation $d \mathrm{da}=0$ ). The second order of the field equation promotes solutions that can be expressed by periodic terms.

Remark 18.For the more simple case we can consider $\nabla \mathrm{h}=0$, as Laplace equation $\nabla^{2} \phi=0$, in flat spaces. The equation (33) and considering a hyperbolic metric for the 4-dimensional flat space, we have an electromagnetic wave equation $\square^{2} \psi=0$.

The connection given in (33) is a meromorphic relative flat connection acting along fibers of the Hitchin mapping

$$
\mathrm{h}: \text { Bun }_{\mathrm{Higgs}} \rightarrow B \text {, }
$$

Furthermore by construction the bundle with connection $(\mathrm{M}, \delta)$, is a Hecke eigen $D$-module with eigen-value $(E, \nabla)$, (as the found in the theorem 4. 1[Bulnes, 2016]) but with respect to an Abelianized version:

$$
{ }^{L} \Phi_{\text {Abelian }}^{1}: D^{b}\left(\operatorname{Bun}_{\mathrm{Higgs}}, O\right) \rightarrow D_{\mathrm{Coh}}\left(\times \mathrm{Bun}_{\mathrm{Higgs}} \times C, O\right),
$$

which is a functor of (22) type. These are defined again for $i=1,2,3, \ldots,(n-1)$, as integral transforms with respect to the trivial local system on the Abelianized Hecke correspondences. Likewise, the superposing of all these Hecke eigen $D$-Modules can determine to the total Hecke category or deformed derived category $\mathcal{H}$.

We can give a graphical representation through 2-dimensional model of (31) considering a re-interpretation as the energy density expressed through the $\mathrm{H}-$ states which can be written using the superposing principle for each connection $\omega_{C}^{\otimes j}$, with $\mathrm{C}$, the curve that describe the corresponding dilaton (photon) in field external presence. Likewise, in Hamilton densities space [Bulnes, 2016] we have the Figure a), considering a Hitchin basis. In the case of a spinor representation the corresponding $\mathrm{H}$ - states can be as spinor waves (Figure b)) which can be consigned in oscillations in the space-time to a microscopic deformation measured [Bulnes, Stropovsvky \& Rabinovich, 2017; Bulnes, 2017b] in $\mathcal{H}$.

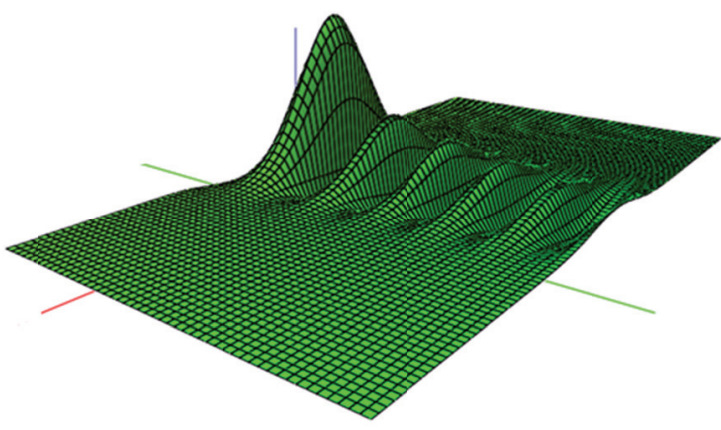

a)

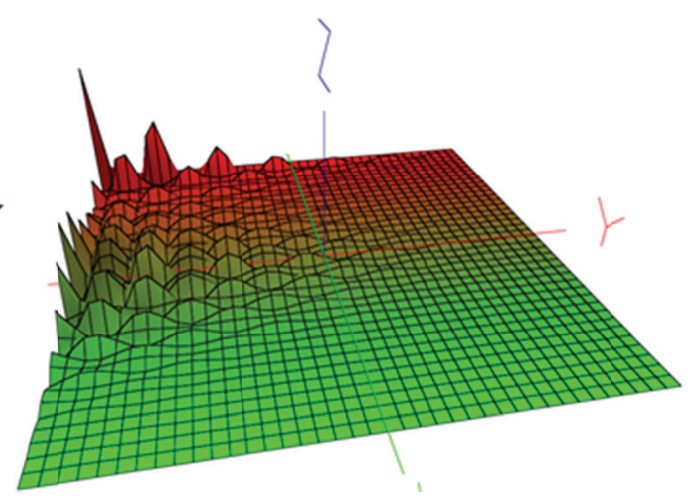

b)

Figure a). Direct sum of $\mathrm{H}$-states to establish the curvature measure by field ramification. b). The waves that are spinor waves which can be consigned in oscillations in the space-time in the presence of curvature to the change of particles spin. This also corresponds to quantum gravity model. Gravitational waves produced by quantum gravity due the $\mathrm{H}$-states on a cylindrical surface. Their propagation is realized on axis $\mathrm{X}$. These gravitational waves are originated for the oscillations in the space-time-curvature/spin (that is to say using causal fermions systems [Bulnes, Stropovsvky \& Rabinovich, 2017]) 


\section{Conclusions}

The result obtained is a simple and linear way for express a deformed category (Hecke category type) $\mathcal{H}$, in components of the hypercohomology obtained for the solutions of the field equations, at least, to the harmonic case, using $\mathrm{H}$-states as Hamiltonian manifold densities. This last using the Hitchin mapping and the characterization of a motives category to the geometrical objects required in the field theory and corresponding to the connections that define its ramifications.

\section{Acknowledgements}

${ }^{*} \mathrm{ITT}_{\mathrm{E}} \mathrm{X}$ Transcription Assistant. Rocio Cayetano

\section{References}

Bulnes, F. (2013). Geometrical Langlands Ramifications and Differential Operators Classification by Coherent $D$-Modules in Field Theory. Journal of Mathematics and Systems Science, 3(10), 491-507.

Bulnes, F. (2016). Integral Geometry Methods in the Geometrical Langlands Program. SCIRP, USA.

Bulnes, F. (2017a). Extended d- Cohomology and Integral Transforms in Derived Geometry to QFT-equations Solutions using Langlands Correspondences. Theoretical Mathematics and Applications, 7(2), 51-62.

Bulnes, F. (2017b). Detection and Measurement of Quantum Gravity by a Curvature Energy Sensor: H-States of Curvature Energy. In D. Uzunov (Ed.), Recent Studies in Perturbation Theory (pp. 169-189), InTech. https//doi.org/ $10.5772 / 68026$

Bulnes, F., Stropovsvky, Y., \& Rabinovich, I. (2017). Curvature Energy and Their Spectrum in the Spinor-Twistor Framework: Torsion as Indicium of Gravitational Waves. Journal of Modern Physics, 8(10), 1723-1736. https//doi.org/10.4236/jmp.2017.810101

Bulnes, F. (2018). Cycles Cohomology and Geometrical Correspondences of Derived Categories to Field Equations. JOURNAL OF ADVANCES IN MATHEMATICS, 14(2), 7880-7892. https://doi.org/10.24297/jam.v14i2.7581

Bulnes, F. (2020a). Geometrical Motives Categories to Determine Co-Cycles as Solutions in Field Theory. Theoretical Mathematics and Applications, 10(2), 15-31.

Bulnes, F. (2020b). Derived Tensor Products and Their Applications. In F. Bulnes (Ed.), Advances in Tensor Analysis and their Applications (pp. 87-102), London, United Kingdom: IntechOpen, Intech. https//doi.org/10.5772/intechopen.92869

Deligne, P. (1994). Structures de Hodge mixtes relles, Motives (Seattle, WA, 1991), Part 1: Proceedings of Symposia in Pure Mathematics, 55 (pp. 509-514) Providence, RI: American Mathematical Society.

Donagi, R., \& Pantev, T. (2010) Lectures on the geometric Langlands conjecture and non-abelian Hodge theory, Philadelphia, PA 19104-6395: University of Pennsylvania, Department of Mathematics.

Griffiths, P. (1968). Periods of integrals on algebraic manifolds I (Construction and Properties of the Modular Varieties). American Journal of Mathematics, 90(2), 568-626. https//doi.org/10.2307/2373545

Mac Lane, S. (1971). Categories for the working mathematician. Springer-Verlag, New York, 1971.

MacPherson, R., Beilinson, A., \& Schechtman, V. (1987). Notes on motivic cohomology. Duke Math. J., 54, 679-710.

Mazza, C., Voevodsky, V., \& Weibel, C. (Editors) (2006). Lecture Notes on Motivic Cohomology, AMS, Clay Mathematics Institute Vol. 2, Cambridge MA, USA.

Milne, J. S. (1980). Étale cohomology, Princeton, N.J: Princeton University Press, ISBN 0-691-08238-3.

Morel, F., \& Voevodsky, V. (1999). A1-homotopy theory of schemes. Publications Mathématiques de l'IHÉS, 90(90), 45-143. https//doi.org/10.1007/BF02698831

Weibel, C. (1994). An introduction to homological algebra. Cambridge University Press, Cambridge.

Quillen, D. (1973). Higher algebraic K-theory. I. Algebraic K-theory, I: Higher K-theories: Proceedings Conf., Battelle Memorial Inst., Seattle, Wash., 1972. Lecture Notes in Math. 341. (pp. 85-147). Berlin, New York: Springer-Verlag. https//doi.org/10.1007/BFb0067053

Saavedra-Rivano, N. (1972). Categories Tannakiennes. Lecture Notes in Mathematics, Vol. 265, Springer-Verlag, Berlin, 1972 (French). MR0338002 (49 \#2769).

Shin, S. W. (2005) Gröthendieck function-sheaf correspondence. Massachusetts, USA: lecture notes from the Harvard seminar on geometric class field theory. 
Voevodsky, V. (2000). Triangulated categories of motives over a field. Cycles, transfers, and motivic homology theories, $143,188-238$.

\section{Copyrights}

Copyright for this article is retained by the author(s), with first publication rights granted to the journal.

This is an open-access article distributed under the terms and conditions of the Creative Commons Attribution license (http://creativecommons.org/licenses/by/4.0/). 